\title{
Synchronization of period-doubling oscillations in vascular coupled nephrons
}

\author{
Laugesen, Jakob Lund; Mosekilde, Erik; Holstein-Rathlou, N. -H.
}

\section{Published in:}

Chaos

Link to article, DOI:

$10.1063 / 1.3641828$

Publication date:

2011

Document Version

Publisher's PDF, also known as Version of record

Link back to DTU Orbit

Citation (APA):

Laugesen, J. L., Mosekilde, E., \& Holstein-Rathlou, N. -H. (2011). Synchronization of period-doubling oscillations in vascular coupled nephrons. Chaos, 21(3), -. https://doi.org/10.1063/1.3641828

\section{General rights}

Copyright and moral rights for the publications made accessible in the public portal are retained by the authors and/or other copyright owners and it is a condition of accessing publications that users recognise and abide by the legal requirements associated with these rights.

- Users may download and print one copy of any publication from the public portal for the purpose of private study or research.

- You may not further distribute the material or use it for any profit-making activity or commercial gain

- You may freely distribute the URL identifying the publication in the public portal

If you believe that this document breaches copyright please contact us providing details, and we will remove access to the work immediately and investigate your claim. 


\section{AIP Chaos}

An Interdisciplinary Journal of Nonlinear Science

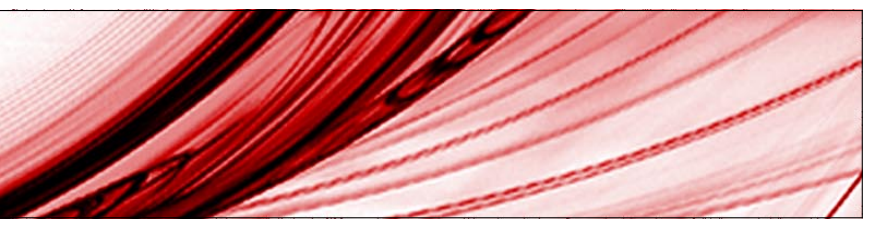

\section{Synchronization of period-doubling oscillations in vascular coupled nephrons}

J. L. Laugesen, E. Mosekilde, and N.-H. Holstein-Rathlou

Citation: Chaos 21, 033128 (2011); doi: 10.1063/1.3641828

View online: http://dx.doi.org/10.1063/1.3641828

View Table of Contents: http://chaos.aip.org/resource/1/CHAOEH/v21/i3

Published by the American Institute of Physics.

\section{Related Articles}

Making human enamel and dentin surfaces superwetting for enhanced adhesion Appl. Phys. Lett. 99, 193703 (2011)

The effects of inhomogeneous boundary dilution on the coating flow of an anti-HIV microbicide vehicle Phys. Fluids 23, 093101 (2011)

A microdevice for the creation of patent, three-dimensional endothelial cell-based microcirculatory networks Biomicrofluidics 5, 034115 (2011)

Clinical diagnostic of pleural effusions using a high-speed viscosity measurement method J. Appl. Phys. 110, 034701 (2011)

Rapid formation of size-controlled three dimensional hetero-cell aggregates using micro-rotation flow for spheroid study

Biomicrofluidics 5, 034105 (2011)

\section{Additional information on Chaos}

Journal Homepage: http://chaos.aip.org/

Journal Information: http://chaos.aip.org/about/about_the_journal

Top downloads: http://chaos.aip.org/features/most_downloaded

Information for Authors: http://chaos.aip.org/authors

\section{ADVERTISEMENT}

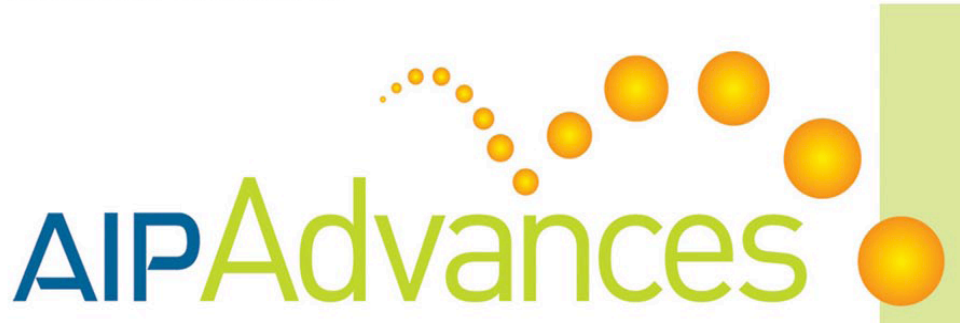

Submit Now

Explore AIP's new

open-access journal

Article-level metrics now available

Join the conversation!

Rate \& comment on articles 


\title{
Synchronization of period-doubling oscillations in vascular coupled nephrons
}

\author{
J. L. Laugesen, ${ }^{1, a)}$ E. Mosekilde, ${ }^{1, b)}$ and N.-H. Holstein-Rathlou ${ }^{2, c)}$ \\ ${ }^{1}$ Department of Physics, The Technical University of Denmark, 2800 Lyngby, Denmark \\ ${ }^{2}$ Department of Biomedical Sciences, University of Copenhagen, 2200 Copenhagen N, Denmark
}

(Received 10 September 2010; accepted 31 August 2011; published online 29 September 2011)

\begin{abstract}
The mechanisms by which the individual functional unit (nephron) of the kidney regulates the incoming blood flow give rise to a number of nonlinear dynamic phenomena, including perioddoubling bifurcations and intra-nephron synchronization between two different oscillatory modes. Interaction between the nephrons produces complicated and time-dependent inter-nephron synchronization patterns. In order to understand the processes by which a pair of vascular coupled nephrons synchronize, the paper presents a detailed analysis of the bifurcations that occur at the threshold of synchronization. We show that, besides infinite cascades of saddle-node bifurcations, these transitions involve mutually connected cascades of torus and homoclinic bifurcations. To illustrate the broader range of occurrence of this bifurcation structure for coupled period-doubling systems, we show that a similar structure arises in a system of two coupled, non-identical Rössler oscillators. @ 2011 American Institute of Physics. [doi:10.1063/1.3641828]
\end{abstract}

Besides their system's nature, by which we refer to the enormous number of interacting feedback regulations that control the biological processes, living organisms are characterized by their sustained and self-generated activity. This activity, ranging from genetic clocks over the complex bursting and spiking dynamics by which the cells organize their internal processes and communicate with one another, to the pacemaker activity and rhythms of the brain, arises through instabilities and nonlinear dynamic phenomena. Regulation of the blood flow to the individual functional unit (nephron) of the kidney involves mechanisms that produce self-sustained oscillations, synchronization of different oscillatory modes in the individual nephron as well as period-doubling transitions. Moreover, adjacent nephrons interact with one another through signals that propagate along their connecting blood vessels. This interaction causes the nephrons to synchronize their blood flow regulation in a complex spatial pattern that reflects the underlying structure of blood vessels but also shows temporal variations, that appear to play an integrated part in overall kidney regulation. The purpose of the present paper is to examine the processes involved in the synchronization and desynchronization of neighboring nephrons. We show that these transitions involve an interesting new structure of interconnected bifurcations. More precisely, we find that the transition from quasiperiodicity and nonsynchronized chaos to synchronized periodic dynamics besides a saddle-node bifurcation also involves the combination of a torus bifurcation and a homoclinic bifurcation. Moreover, these bifurcations are organized in a characteristic structure such that a new set of bifurcation curves arise for each period-doubling bifurcation of the

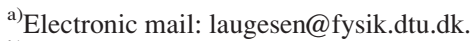

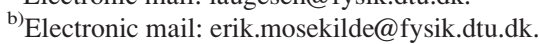

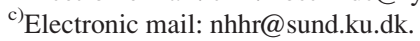

individual nephrons. We finally show that a similar structure occurs in a pair of coupled, non-identical Rössler systems.

\section{INTRODUCTION}

Synchronization and other nonlinear dynamic phenomena play an essential role in the regulation and function of normal physiological systems. ${ }^{1}$ It is well-known, for instance, that the beating of the heart and the respiratory cycle adjust to one another and temporarily may lock into states with rational ratios (such as 1:3, 1:4, or 2:7) between the two periodicities. ${ }^{2}$ At the cellular level, one can observe how neighboring $\beta$-cells in a pancreatic islet synchronize their electrophysiological bursting dynamics and, thereby also, their rapid pulses of insulin secretion. ${ }^{3}$ Fluorescent microscopy studies ${ }^{4}$ have shown how smooth muscle cells in vascular resistance vessels at low activation display asynchronous wavelike fluctuations in their cytoplasmic calcium concentrations. As the muscular activation increases, a growing number of cells are recruited into a synchronized state, thereby producing rhythmic contractions of the vessel (vasomotion) with frequencies in the $100-200 \mathrm{mHz}$ regime.

In other cases, such as Parkinson's tremor, the development of a disease may be ascribed to the transition of a cluster of cells (in the brain) from a state of relatively independent spiking into a state of strong synchronization. Patients who cannot be treated through medication may often be helped by deep brain electrical stimulation designed to de-synchronize the cellular dynamics ${ }^{5}$ or by selective modulation of brain rhythms by means of multi-electrode arrays. ${ }^{6}$ As another example, Kronenberg et al. ${ }^{7}$ have demonstrated how the characteristic variations in blood flow and body temperature observed during menopausal hot flashes are 
associated with synchronization of a number of hormonal secretion processes.

Let us finally mention that one can sometimes take advantage of the synchronization of cellular processes to improve the treatment of a disease. The individual cells in the body dispose of a circadian timing system. These cellular clocks are synchronized by hypothalamic pacemaker signals, and this allows the circadian clock to coherently modulate hormonal secretion, cellular metabolism, and many other physiological processes. In particular, the circadian timing system modulates both cellular proliferation rates and the toxicity of many drugs and, as demonstrated by Lévi et al., ${ }^{8}$ this effect is strong enough for an $8 \mathrm{~h}$ shift in administration time to produce an eightfold increase in the tolerability of more than 30 anti-cancer drugs.

As part of an effort to understand the relation between hypertension and kidney function, we have long been engaged in a study of nephron autoregulation, i.e., of the mechanisms by which the individual functional unit of the kidney regulates the incoming blood flow in response to variations in the arterial pressure. $^{9-11}$ This regulation involves two different mechanisms: A myogenic mechanism that reacts directly to changes in the arterial pressure, and a socalled tubuloglomerular feedback (TGF) mechanism that responds to signals from specialized cells (the macula densa cells) near the terminal part of the loop of Henle.

The myogenic mechanism depends on an inherent propensity of the smooth muscle cells in the arteriolar wall to contract in response to an increasing pressure difference across the vascular wall. ${ }^{12,13}$ This contraction causes the flow resistance to increase and, thereby, leads to a lower glomerular pressure and a reduced rate of filtration. The TGF mechanism, on the other hand, depends on a response from the macula densa cells to changes in the salt concentration of the tubular fluid. A high rate of glomerular filtration leads to a faster flow through the loop of Henle, to incomplete reabsorption of salt from the tubular fluid, rising salt concentrations at the macula densa, and a signal to the smooth muscle cells in the arteriolar wall to contract, thus causing the rate of filtration to decline.

The TGF mechanism is a negative feedback. However, as demonstrated in experiments on rats, ${ }^{9,10}$ this mechanism tends to be unstable and to produce large amplitude selfsustained oscillations in the tubular pressures and flows with periods in the 30-40 s range. The instability in the feedback regulation and the relatively long periodicity of the oscillations are directly related to the time of $12-15 \mathrm{~s}$ that it takes for the tubular fluid to pass the loop of Henle. ${ }^{14}$

The myogenic (or vasomotoric) mechanism also produces oscillations in the afferent arteriolar resistance as the muscular activation increases. As mentioned above, ${ }^{4,12}$ the transition to self-sustained oscillations in this case takes place through synchronization of the cytoplasmic $\mathrm{Ca}^{2+}$ waves in the individual cells. The period of the vasomotoric oscillations in the afferent arterioles of the kidney is typically 6-8 s, or approximately a factor 5 shorter than the period of the TGF oscillations.

The two regulatory mechanisms both work through activation of the smooth muscle cells in the arteriolar wall. This allows the oscillatory modes to interact and to produce frequency-locking with typical locking ratios of 1:4, 1:5, and 1:6. ${ }^{15,16}$ Nephron pressure and flow regulation involves a number of additional nonlinear relations, including the nonlinear feedback characteristic for the TGF mechanism, ${ }^{18}$ the nonlinear static strain-stress relation for the arteriolar wall, ${ }^{19}$ and the nonlinear relation between the concentration and osmotic pressure of protein in the blood. As a result, episodes of period-2 dynamics can be observed in about $50 \%$ of the experimental time traces for the proximal tubular pressure in normotensive rats. ${ }^{20}$

Figure 1 shows an example of such a time series. The mean tubular pressure in this experiment is about $7 \mathrm{mmHg}$. But this pressure is strongly modulated by relatively slow TGF-mediated oscillations with an amplitude of about 1-2
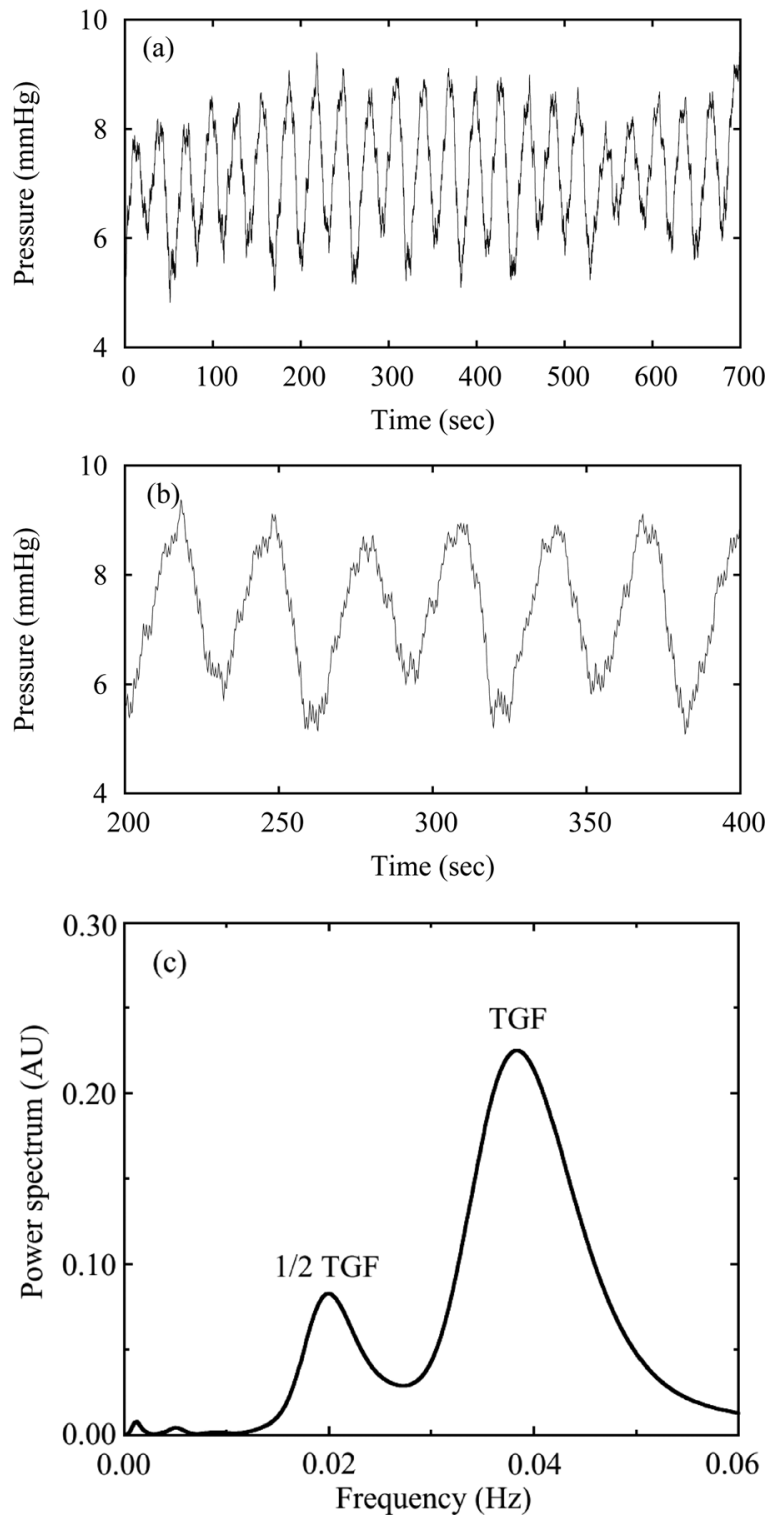

FIG. 1. Experimentally observed time series for the proximal tubular pressure in a normotensive rat. (a) Large amplitude oscillations produced by instability in the TGF. (b) Alternating deep and shallow minima signaling period-2 dynamics. (c) Power spectrum showing the main TGF peak at $40 \mathrm{mHz}$ together with a subharmonic component at $20 \mathrm{mHz}$. For hypertensive rats, the tubular pressure oscillations are typically chaotic. Reproduced with permission from Interface Focus 1, 132 (2011). 
$\mathrm{mmHg}$. The faster myogenic oscillations manifest themselves as a ripple on top of the TGF oscillations. Closer examination of the time trace in Fig. 1(a) reveals an episode of period-2 dynamics lasting approximately from time $200 \mathrm{~s}$ to time 500 s. This part of the time trace is amplified in Fig. 1(b), where the tubular pressure oscillation is seen to alternate between shallow and deep minima. The corresponding power spectrum, shown in Fig. 1(c), also provides evidence for the presence of subharmonic components in the pressure variation.

The nephrons are organized in a tree structure around a common blood supply, often such that pairs (or triples) of nephrons share part of an afferent arteriole. ${ }^{21}$ This allows the functional units to interact with one another, ${ }^{10}$ both via a simple displacement of blood from one nephron to its neighbors as the first nephron reduces its incoming blood flow (hemodynamic coupling) and via signals of muscular activation that travel from nephron to nephron along the blood vessels (vascular propagated coupling). ${ }^{22}$ These two mechanisms tend to synchronize the pressure oscillations of the interacting nephrons, typically such that either in-phase or antiphase operation is observed. ${ }^{23,24}$

The purpose of the present paper is to examine the transitions in and out of synchrony that occur through interaction between a pair of neighboring nephrons. The classical theory of synchronization ${ }^{25}$ considers a system of two interacting limit cycle oscillators (or a single oscillator subjected to an external periodic forcing). This theory explains the synchronization transition in terms of a saddle-node bifurcation in which a stable (node) and an unstable (saddle) cycle of the same periodicity appear (or disappear) on the surface of a two-dimensional torus. As the frequency mismatch (detuning) is changed for fixed coupling parameter, the oscillators display regions of synchronization (Arnol'd tongues) for all possible rational ratios between the two periodicities. For increasing coupling parameter, the width of these tongues (i.e., the range of detuning parameters for which synchronization takes place) generally increases.

The classical theory has successfully been used to describe a broad range of different synchronization phenomena in physiology as well as in other fields of science. ${ }^{1,2}$ However, new phenomena can be expected to arise in the synchronization of more complicated oscillatory modes. It is known, for instance, that a period-doubling cascade that unfolds along the edge of a resonance zone may display an unusual organization and a specific scaling behavior, referred to as cyclic (or C-type) criticality. ${ }^{26,27}$ In studies of periodically forced period-doubling systems, one observes that the stable and unstable resonance cycles generated at the edge of the synchronization regime undergo interconnected cascades of period-doubling bifurcations and that each period doubling leads to the formation of a new pair of saddle-node bifurcation curves along the edges of the resonance zone. ${ }^{27,28}$ Moreover, these saddle-node bifurcations accumulate to finally define the transition between phase synchronized chaos and non-synchronous chaos. Outside the resonance regions, one can observe the phenomenon of ergodic torus-doubling as discovered originally by Arnéodo et al. ${ }^{29}$ and by Kaneko, ${ }^{30}$ and as recently demonstrated in practice by Sekikawa et al. ${ }^{31}$ for an electronic oscillator system.
The present analysis shows that a new and even more complicated structure arises in connection with the synchronization of two vascular coupled nephrons. In particular, we find that the transition from synchronized periodic dynamics to asynchronous chaotic dynamics besides a cascade of saddle-node bifurcations involves the combination of cascades of torus and homoclinic bifurcations. To illustrate the broader range of occurrence of this transition, we demonstrate in Sec. IV that a similar structure arises in a system of two coupled Rössler oscillators.

\section{MODEL STRUCTURE}

Over the years, we have developed a number of different models of the regulation of the afferent blood flow to the individual nephron, ${ }^{32-34}$ each emphasizing a specific aspect of the problem such as the absorption of water and salts along the loop of Henle ${ }^{32}$ or the interaction between the macula densa cells and the smooth muscle cells in the arteriolar wall. ${ }^{34}$ In the present paper, we will use the model developed by Barfred et al..$^{33}$ This model integrates the most essential aspects of nephron autoregulation into a consistent picture and is, due to its relatively simple structure, particularly useful for detailed bifurcation studies. The same model has been applied in a number of earlier studies of nephron-nephron interaction. ${ }^{23,35}$

The first component in the model is a conservation equation,

$$
\frac{d P_{t}}{d t}=\frac{1}{C_{\text {tub }}}\left[F_{\text {filt }}-F_{\text {reab }}-F_{H e n}\right]
$$

that relates the changes in the proximal tubular pressure $P_{t}$ to the rate of glomerular filtration $F_{\text {filt }}$, the absorption that takes place in the proximal tubule $F_{\text {reab }}$, and the flow into the loop of Henle $F_{H e n}$. $C_{t u b}$ denotes the elastic compliance of the proximal tubule. This equation is supported by a number of algebraic equations that determine first the rate of glomerular filtration,

$$
F_{\text {filt }}=\left(1-H_{a}\right)\left(1-\frac{C_{a}}{C_{e}}\right)\left(\frac{P_{a}-P_{g}}{R_{a}}\right),
$$

in terms of the arterial pressure $P_{a}$, the glomerular pressure $P_{g}$, and the flow resistance $R_{a}$ of the afferent arteriole, and second the flow into the loop of Henle,

$$
F_{\text {Hen }}=\frac{P_{t}-P_{d}}{R_{H e n}},
$$

in terms of the proximal tubular pressure $P_{t}$, the distal tubular pressure $P_{d}$, and the tubular flow resistance $R_{H e n}$. Here, $H_{a}$ is the afferent hematocrit (i.e., the fraction of the blood volume that the blood cells occupy), and $C_{a}$ and $C_{e}$ are the concentrations of protein in the afferent and the efferent blood, respectively. The factor involving the two protein concentrations expresses the fact that, since protein is retained in the blood, the change in blood volume due to filtration is (inversely) related to the increase in protein concentration from the afferent to the efferent side of the glomerulus. 
To determine the efferent protein concentration $C_{e}$, we make use of the assumption that before the blood leaves the glomerulus its protein osmotic pressure $P_{\text {osm }}$ balances the hydrostatic pressure difference $\left(P_{g}-P_{t}\right)$ between the glomerulus and the proximal tubule. This gives

$$
C_{e}=\frac{1}{2 b}\left[\sqrt{a^{2}+4 b\left(P_{g}-P_{t}\right)}-a\right]
$$

where $a$ and $b$ are experimentally determined parameters that relate protein osmotic pressure $P_{\text {osm }}$ and concentration C,

$$
P_{\text {osm }}=a C+b C^{2} \text {. }
$$

The glomerular pressure $P_{g}$ is determined by adding the pressure drop across the efferent arteriolar resistance $R_{e}$ to the venous pressure $P_{v}$, i.e.,

$$
P_{g}=P_{v}+R_{e}\left(\frac{P_{a}-P_{g}}{R_{a}}-F_{f i l t}\right)
$$

where the terms between the parentheses represent the blood flow through the efferent arteriole. The TGF mediated modulation of the glomerular filtration is expressed by means of a sigmoidal relation between the activation $\psi$ of the smooth muscle cells in the afferent arteriolar wall and the tubular flow $X_{3}$ at the macula densa, ${ }^{36}$

$$
\psi=\psi_{\max }-\frac{\psi_{\max }-\psi_{\min }}{1+\exp \left\{\alpha\left(3 X_{3} / T F_{H e n, 0}-S\right)\right\}} .
$$

Here, $\psi_{\max }$ and $\psi_{\text {min }}$ denote maximal and minimal values of the muscular activation. $\alpha$ and $S$ are parameters that determine, respectively, the slope and the displacement of the characteristic along the flow axis, $F_{H e n, 0}$ is a normalization value for the Henle flow, and $T$ is the delay associated with the flow of fluid through the loop of Henle.

The activation $\psi$ (also called the tone) represents a welldefined concept in muscular physiology. In this connection, it is of interest to note that smooth muscle cells are capable of maintaining a certain tone in their resting state. ${ }^{17}$ The form of the feedback characteristic (7) can be obtained from open-loop experiments in which the rate of glomerular filtration is measured as a function of the rate at which artificial tubular fluid is infused into the loop of Henle while flow through the proximal tubule is prevented by a wax seal. ${ }^{18,36}$ In our dynamic model, we replace the Henle flow in the feedback characteristic (7) by a delayed version $3 X_{3} / T$ of this flow obtained from the simple delay structure,

$$
\begin{aligned}
\frac{d X_{1}}{d t} & =F_{H e n}-\frac{3}{T} X_{1} \\
\frac{d X_{2}}{d t} & =\frac{3}{T}\left(X_{1}-X_{2}\right) \\
\frac{d X_{3}}{d t} & =\frac{3}{T}\left(X_{2}-X_{3}\right) .
\end{aligned}
$$

The myogenic modulation of the afferent arteriolar flow resistance is modeled by a second order differential equation for the normalized arteriolar radius $r$ of the form,

$$
\frac{d^{2} r}{d r^{2}}+d \frac{d r}{d t}+\Omega\left(P_{a v}-P_{e q}\right)=0
$$

in connection with a relation between the arteriolar resistance $R_{a}$ and the normalized radius derived from Poisson's equation,

$$
R_{a}=\left(\beta+(1-\beta) r^{-4}\right) R_{a, 0}
$$

Here, the parameter $d$ is the damping coefficient for the myogenic oscillations, $\Omega$ determines the frequency of these oscillations, $1-\beta$ represents the active part of the arteriolar length, and $R_{a, 0}$ is the equilibrium value of the afferent arteriolar resistance.

As described above, the myogenic (or vasomotoric) oscillations change character with increasing muscular activation as more and more cells are recruited into a synchronized state. In the form we use the equations here, the myogenic oscillations are assumed to be damped $(d>0)$, but they are continuously excited by the TGF activity. This excitation is mediated through variations in the difference $P_{a v}-P_{e q}$ between the average pressure calculated for the active part of the arteriole,

$$
P_{a v}=\frac{1}{2}\left(P_{a}-\left(P_{a}-P_{g}\right) \beta \frac{R_{a, 0}}{R_{a}}+P_{g}\right),
$$

and the value $P_{e q}$ of this pressure at which the arterial wall is in equilibrium at its present radius and muscular tone.

The steady state reaction of the arteriolar wall to changes in the blood pressure is assumed to consist of a passive, elastic component $P_{e l}(r)$ in parallel with an active muscular component $\psi P_{a c t}(r)$, i.e.,

$$
P_{e q}=P_{e l}(r)+\psi P_{a c t}(r)
$$

where, as before, $\psi$ denotes the degree of muscular activation. The elastic contribution typically takes the form

$$
P_{e l}(r)=P_{1}\left(e^{\alpha_{1}(r-1)}+b_{1}(r-1)\right),
$$

where the parameters $P_{1}, \alpha_{1}$, and $b_{1}$ depend on the size of the arteriole and the tissue in which it is situated. This part of the static strain-stress relationship is dominated by the exponential variation in Eq. (13) that causes the arteriole to resist excessive dilatation. The active part of the strain-stress relation is approximately given by a sigmoidal contribution, ${ }^{19}$

$$
P_{a c t}(r)=P_{2}\left[\frac{1}{1+e^{\alpha_{2}(0.4-r)}}+b_{2}(r+0.9)\right]
$$

that expresses the experimental observation that activation of the smooth muscle cells in the arteriolar wall takes place in a certain range of the transmural pressure (corresponding to a normalized arteriolar radius around its resting value $r=1$ ). At very small pressures, there is no muscular activation, and for large values of the transmural pressure, the activation of the smooth muscle cells again decreases. A detailed derivation of the expression for the active contribution may be found in the work by Feldberg et al. ${ }^{19}$ Most of the 
parameters in the model have been determined from independent measurements. The time delay for the fluid flow through the loop of Henle, for instance, has been obtained from the phase shift between the oscillations in the fluid salt concentration near the macula densa and in the proximal tubular pressure. ${ }^{14}$ Other parameters have been derived from the literature. Values for the different parameters may be found in the original publication. ${ }^{33}$

\section{BIFURCATION STRUCTURE FOR VASCULAR COUPLED NEPHRONS}

The regulatory mechanisms of the individual nephron act as a mechanical high-pass filter to protect the delicate processes that take place in the distal tubule from more lasting variations in the arterial blood pressure. While the myogenic mechanism is active in most tissues, the TGF mechanism is an additional mechanism required for the nephrons to handle the enormous blood flow that the kidneys receive. The response time of the TGF mechanism is restricted by the time it takes the tubular fluid to flow through the loop of Henle, and the system presumably achieves the fastest possible response by adopting an oscillatory mode of regulation. As described in previous work, ${ }^{23,24}$ interaction between adjacent nephrons leads to synchronization of their pressure oscillations. Experimentally, one typically observes that the tubular pressures in a pair of neighboring nephrons oscillate in synchrony for a period of 20-40 min, lose synchrony for a while and then synchronize again.

Interaction between neighboring nephrons takes place via two different mechanisms, a vascularly propagated mechanism $^{22}$ and a simple hemodynamic mechanism. The vascular propagated mechanism makes use of signals that travel from one nephron to its neighbors along the smooth muscle cells in the vascular wall. The amplitude of these signals decay more or less exponentially with distance, but their rate of propagation is quite fast as compared with the distance between the nephrons in terms of the period of the TGF oscillations. The vascular propagated coupling may therefore be considered to be instantaneous. In the present analysis, the coupling is assumed to be symmetric, and it is assumed that a fraction $\gamma=0.05$ of the muscular activation $\left(\psi_{1}\right.$ or $\left.\psi_{2}\right)$ of one arteriole reaches the active region of the other nephron, i.e.,

$$
\begin{aligned}
& \psi_{1}^{*}=\psi_{1}+\gamma\left(\psi_{2}-\psi_{1}\right) \\
& \psi_{2}^{*}=\psi_{2}+\gamma\left(\psi_{1}-\psi_{2}\right)
\end{aligned},
$$

where $\psi_{1}^{*}$ and $\psi_{2}^{*}$ are the muscular activations in the coupled nephron system.

In order to examine the transitions that occur as a couple of nephrons move in and out of synchrony, we shall make use of standard continuation methods. Continuation ${ }^{37}$ is a widely used numerical technique developed to locate and follow periodic solutions as a parameter is changed, to determine bifurcation points and to follow bifurcation curves in phase space under variation of one (or more) parameters. In this way, one can obtain a complete survey of the dynamics of a given system, the bifurcations it undergoes as a parameter is changed, and the overall structure of interconnected bifurcations. In the present study, we have applied the free software available from Doedel et al. ${ }^{38}$

Figure 2 provides a survey of the regions of existence for different behavioral modes in and around the main 1:1 synchronization zone. Parameters of the phase diagram are the delay $T^{(2)}$ in the loop of Henle for nephron-2 and the afferent arteriolar resistance $R_{a, 0}^{(1)}$ for nephron-1. These parameters represent, respectively, the detuning and a built-in asymmetry between the two nephrons. Base case values are $T^{(1)}=16 \mathrm{~s}$ and $R_{a, 0}^{(2)}=2.4 \mathrm{kPa} \cdot \mathrm{s} / \mathrm{nl}$, and these values correspond to a situation in which the individual nephron operates in a state of 1:5 synchronization with respect to its internal modes.

Different shades of blue and green are used to designate the regions of existence of stable low periodic orbits associated with the initial steps in the period-doubling cascade. Dark blue denotes regions of synchronized period-1 dynamics for the TGF oscillations. Light blue denotes regions of synchronized period-2 TGF oscillations, i.e., in these regions both nephrons have undergone a period-doubling bifurcation, but remain synchronized both with respect to the TGF oscillations and with respect to the faster myogenic oscillations. Green denotes regions of synchronized period-4 dynamics. The yellow area to the left in the diagram is a regions of synchronized period-3 dynamics. The red regions represent other regions of synchronized periodic dynamics, including a region with synchronized period-6 dynamics (close to the yellow region of period-3 dynamics) and different windows with period-5 dynamics. Finally, gray denotes regions of phase synchronized chaos while brown and orange denote regions with non-synchronous quasiperiodic and chaotic dynamics, respectively. In the region of phase synchronized chaos, both nephrons display chaotic dynamics, but their phases move in near synchrony. 39,40

Inspection of Fig. 2 allows us to identify a main perioddoubling structure connecting as a (twisted) horseshoe from the top to bottom in the resonance zone and accumulating in

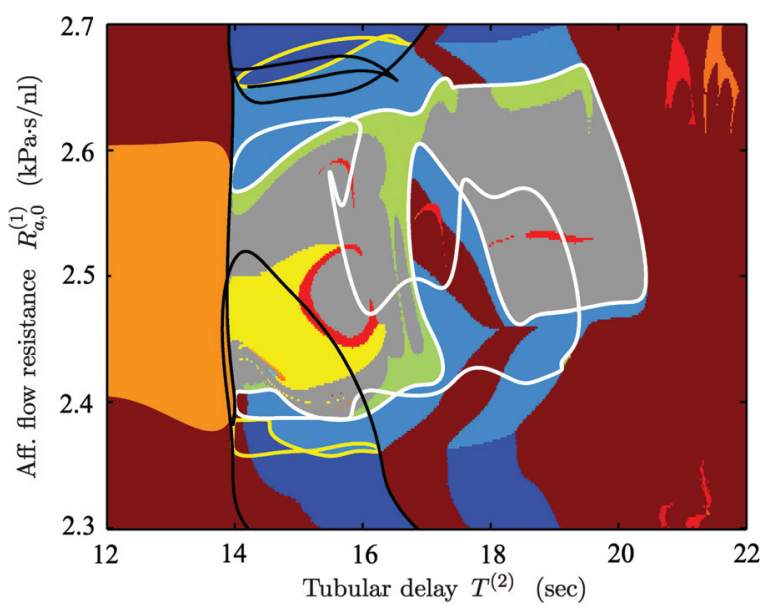

FIG. 2. (Color) Survey of the bifurcation structure in the main synchronization regime for a model of two coupled nephrons. Parameters in the figure are the resting value of the afferent arteriolar flow resistance for one of the nephrons and the delay of the fluid flow through the loop of Henle for the other nephron. The color code refers to the periodicity of the various cycles. The yellow region represents the region of synchronized period-3 dynamics. Reference values for the two parameters are $R_{a, 0}^{(2)}=2.4 \mathrm{kPa} \cdot \mathrm{s} / \mathrm{nl}$ and $T^{(1)}=16.0 \mathrm{~s}$. 
the gray region of phase synchronized chaos around the large yellow window of synchronized period-3 dynamics. The two closed yellow curves are period-doubling curves along which a transition from synchronized period-1 to synchronized period-2 dynamics takes place. Parts of these curves follow the demarcation lines between the dark and light blue regions, indicating that the transition involves a stable period-1 solution. Other parts of the two curves represent period-doubling of the synchronized period-1 saddle. With all its windings, the closed white curve similarly represents the period-doubling curve at which a transition from synchronized period-2 to synchronized period-4 dynamics takes place. Again, parts of the bifurcation curve are seen to follow the demarcation lines between light blue and green regions, indicating that the bifurcation involves the transition from stable period-2 dynamics to stable period-4 dynamics. The black curves, parts of which proceed along the left hand side of the resonance zone in Fig. 2, represent the saddlenode bifurcations in which the resonant period-1 and period2 solutions are born from the quasiperiodic dynamics that exists in the brown region to the left of the zone. As explained below, new saddle-node bifurcation curves are found to emerge along the zone boundary to delineate the regions of existence for higher modes in the synchronized period-doubling cascades.

Along the upper left boundary of the resonance zone, the saddle-node bifurcation curves are seen to follow the demarcation lines between the brown quasiperiodic regime and the dark and light blue regions with period-1 and period-2 dynamics, respectively. This implies that (except for small corners around the points of appearance for the new saddlenode bifurcation curves ${ }^{26-28}$ ) the synchronized periodic modes are born as pairs of stable nodes and singly-unstable saddle cycles. The same is found to be true for the following modes in the period-doubling cascade.

The scenario we have outlined for the upper left edge of the synchronization zone develops in accordance with the picture established for C-type criticality. ${ }^{26,27}$ By contrast, along the lower left part of the zone boundary, the quasiperiodic brown region is seen to cross the saddle-node bifurcation curves and penetrate into the region of synchronized periodic dynamics. In this case, the saddle-node bifurcations do not produce stable resonance cycles, but stabilization of these cycles occurs through some different mechanism and takes place inside the resonance zone. The purpose of the following analysis is first to identify this mechanism and second to show that a similar mechanism can be observed for two coupled, non-identical Rössler oscillators. We shall demonstrate how each pair of period-doubling bifurcations besides a new pair of saddle-node bifurcations to delineate the range of existence of the synchronized periodic modes (as for C-type criticality) also generates a torus bifurcation curve that continues up along the edge of the synchronization zone and marks the stabilization of the periodic resonance dynamics. We shall also demonstrate how this bifurcation structure allows the interacting nephrons to attain a state of phase synchronized quasiperiodic motion.

Figure 3 shows a more detailed representation of the unusual bifurcation structure observed in the lower left corner

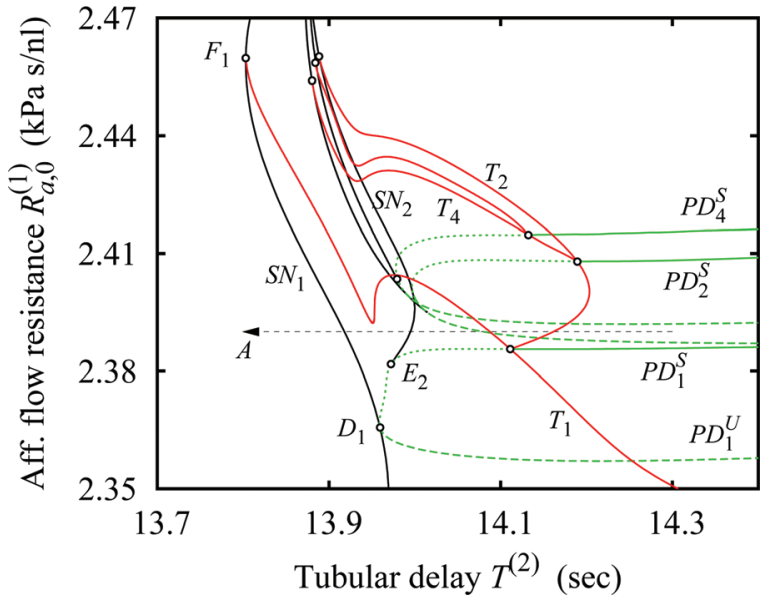

FIG. 3. (Color) Blow-up of the bifurcation structure in the lower left corner of Fig. 2. Saddle-node bifurcation curves are black, period-doubling curves green, and torus bifurcation curves red. Note how the torus bifurcation curves attach to points on the corresponding saddle-node bifurcation curves along the edge of the resonance tongue. This process involves a mechanism by which new torus bifurcation curves are formed alternately to the right and the left of the former torus bifurcation curve.

of the bifurcation diagram in Fig. 2. Here, period-doubling curves are green, and saddle-node bifurcation curves are black. In addition to these curves, we have also drawn (red) a set of torus bifurcation curves in which a periodic solution loses (or gains) its stability as a pair of complex conjugated multipliers crosses out of (or into) the unit circle in the complex plane.

We immediately identify the saddle-node bifurcation curve $S N_{1}$ to the left in the figure. $S N_{1}$ is tangent to the first period-doubling curve $P D_{1}$ at the point $D_{1} . P D_{1}^{S}$ and $P D_{1}^{U}$ denote the first period-doubling curves for the stable and the unstable period-1 cycle, respectively. The saddle-node bifurcation $S N_{1}$ serves to delineate the range of existence of period-1 resonant cycles. However, when the point of operation crosses this saddle-node bifurcation curve into the resonance region below the point $D_{1}$, it is found to give birth to a pair of doubly and a triply unstable saddle solutions rather than, as normally expected, to a saddle and a stable node cycle. A second saddle-node bifurcation curve $S N_{2}$, delineating the range of existence for period-2 resonance cycles, emerges from the point $E_{2}$ on the unstable branch of the first period-doubling bifurcation curve. Again, a pair of double and triple unstable period- 2 saddle cycles is produced when the point of operation crosses this saddle-node bifurcation. As well as we can follow the structure, the same picture repeats itself all the way up along the period-doubling cascade.

The first torus bifurcation curve $T_{1}$ is supported by the point $F_{1}$ on the saddle-node bifurcation curve $S N_{1}$. From here, with a couple of turns, $T_{1}$ proceeds down along the saddle-node bifurcation curve, to finally return to the upper part of the bifurcation diagram (not shown). At the point where $T_{1}$ intersects the stable branch $P D_{1}^{S}$ of the first perioddoubling curve, a new torus bifurcation curve $T_{2}$ emerges, and at the point where this torus bifurcation curve intersects the stable branch $P D_{2}^{S}$ of the second period-doubling curve a third torus bifurcation curve $T_{4}$ arises. In accordance with 
our numerical calculations, this cascade of torus bifurcation curves continues along with the cascade of period-doubling transitions.

It is interesting to note how the torus bifurcation curves appear alternately to the right and the left of the former torus bifurcation curve. This resembles the characteristic alternating features of C-type criticality. ${ }^{26,27}$ Note also how the torus bifurcation curves attach to points on the corresponding saddle-node bifurcation curves along the edge of the resonance zone. From each of these points, a curve of homoclinic bifurcations stretches along the left hand side of the torus bifurcation curve all the way down to the point, where the torus bifurcation curve emerges in the crossing between the former torus bifurcation curve and the stable branch of the corresponding period-doubling curve. We conclude that the system also displays an infinite set of homoclinic bifurcation curves.

Figure 4 shows a scan through the boundary of the synchronization regime along the direction $\mathrm{A}$ in Fig. 3 for $R_{a, 0}^{(1)}=2.39 \mathrm{kPa} \cdot \mathrm{s} / \mathrm{nl}$. Following the scan from right to left, we start in a situation where the coupled nephrons displays a stable (and a singly unstable) synchronized period-2 solution. These solutions correspond to the resonance modes, in which one would normally expect to find in the synchronization region. However, as we move to the left, rather than undergoing a saddle-node bifurcation, the stable period- 2 cycle undergoes a torus bifurcation at $T_{2}$, producing a period-2 torus with quasiperiodic dynamics and a (doubly) unstable period-2 focus cycle. As the point of operation moves further to the left, the period-doubled torus undergoes the above mentioned homoclinic bifurcation at $\mathrm{H}_{2}$, and non-synchronous chaotic dynamics arises. Periodic states still exist, but only in the form of saddle and unstable focus cycles. The saddle-node bifurcation $\mathrm{SN}_{2}$ falls far to the left of the bifurcation scan and
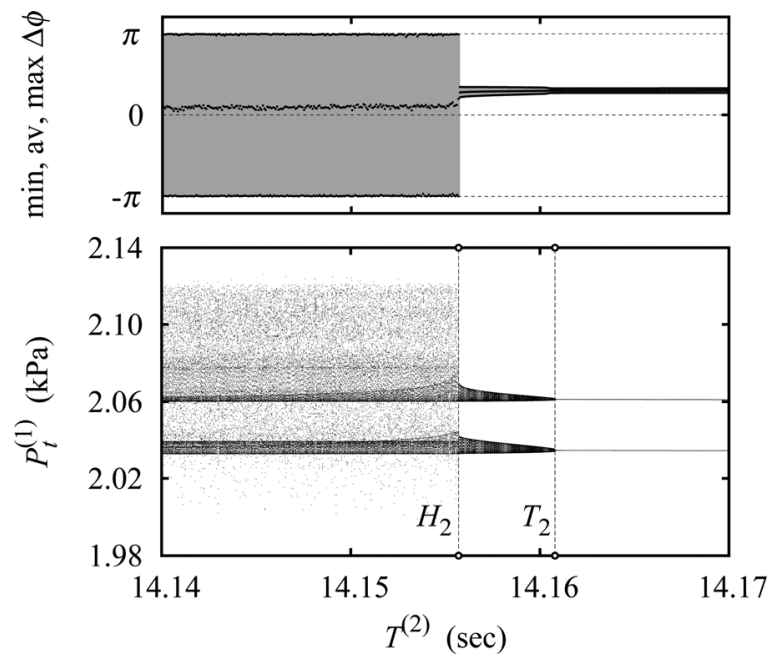

FIG. 4. One-dimensional bifurcation diagram obtained by scanning through the boundary of the resonance zone along the direction $A$ in Fig. 3 for $R_{a, 0}^{(1)}=2.39 \mathrm{kPa} \cdot \mathrm{s} / \mathrm{nl}$. Read from right to left the diagram illustrates the transition from stable synchronized period-2 dynamics to non-synchronous chaos through a torus bifurcation followed by a homoclinic bifurcation. In the upper panel, we have plotted the average phase difference between the two oscillators together with the smallest and largest phase difference observed during a very long simulation. Note that this phase difference remains restricted in the region of torus dynamics. is not directly involved in the transition from stable periodic dynamics to non-synchronous behavior.

Figures 5(a)-5(c) show phase portraits of the stable period-2 solution, the period-2 torus, and the nonsynchronous chaotic attractor, respectively. It is interesting to note that the torus bifurcation does not destroy the phase synchronization of the pressure and flow variations between the two nephrons. This is illustrated in the panel displayed above the bifurcation diagram in Fig. 4. Here, the average phase difference between the two nephrons is plotted as a function of the bifurcation parameter (the delay in the loop of Henle for nephron-2). In this calculation, the phase difference $\Delta \phi$ is defined as the phase of the delay variable $X_{3}^{(2)}$ each time the coupled system crosses a Poincaré section at $X_{3}^{(1)}=1.0 \mathrm{nl}$. The phase of $X_{3}^{(2)}$ is defined as the trigonometric angle $\phi_{2}=\tan ^{-1}\left(X_{3, n}^{(2)} / X_{2, n}^{(2)}\right)$, where the index $n$ denote the $n$ 'th crossing in the Poincaré section. The delay variables are particularly useful for this analysis because their myogenic components are very weak.

To the right of the torus bifurcation $T_{2}$, the synchronized period-2 dynamics involves a small period- 2 component in the phase of the second nephron relative to the phase of the first nephron. This phase modulation increases a little in the quasiperiodic state to the left of $T_{2}$, but remains much smaller than $2 \pi$. Only the homoclinic bifurcation $H_{2}$ destroys the phase synchronization between the two nephrons as indicated by the abrupt jump of the range of observed phase differences to $\pm \pi$.

The torus bifurcation gives birth to a new, small amplitude and slowly varying mode in the system, but this mode has no direct relation, neither to the TGF nor to the myogenic
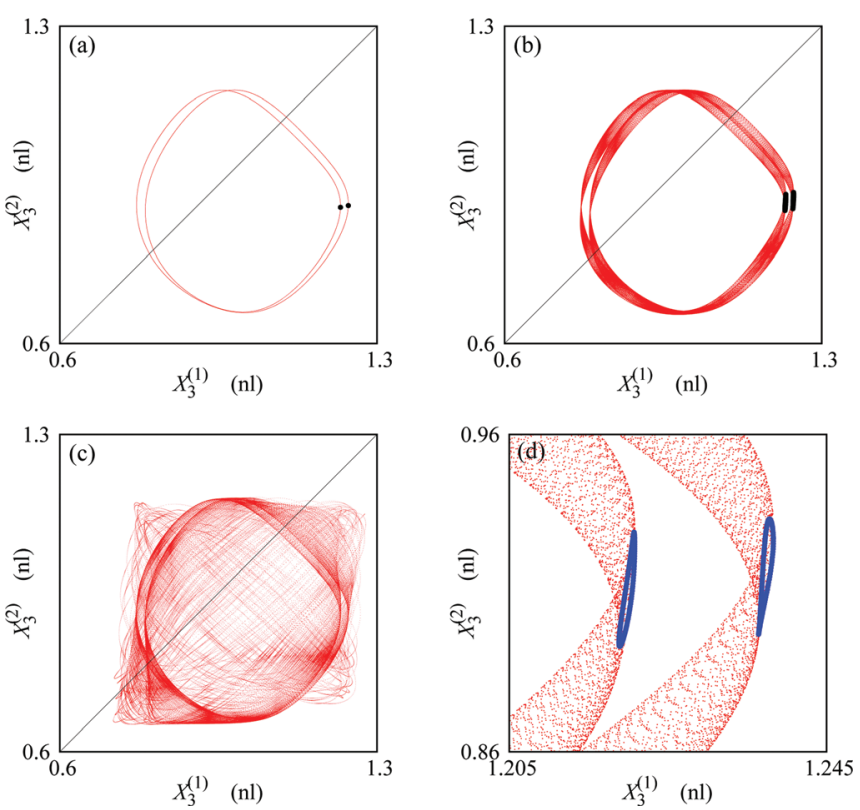

FIG. 5. (Color) Phase portraits of the solutions observed in the scan of Fig. 4. As given by Eq. (9), the delay variables $X_{3}^{(1)}$ and $X_{3}^{(2)}$ represent the fluid flow near the end of the loop of Henle for the two nephrons. (a) Stable synchronized period-2 solution. (b) Synchronized quasiperiodic solution. (c) Nonsynchronous chaotic solution. (d) Blow-up of part of the phase portrait in (b). The blue dots show the phase of the second oscillator whenever the first oscillator passes the Poincaré section $X_{3}^{(1)}=1.0 \mathrm{nl}$. 
oscillations. We conclude that, while maintaining internal synchrony between their myogenic and TGF-mediated oscillations, the two nephrons in their relative dynamics move in a phase-synchronized quasiperiodic state in which the phase difference varies, but the variations remain much smaller than $2 \pi$. A number of related phenomena have recently been encountered by Anishchenko et al ${ }^{41,42}$ in investigations of systems of interacting two-mode electronic generator circuits. At the present, however, it appears that many questions related to the synchronization of multimode oscillators remain unsolved.

\section{COUPLED, NON-IDENTICAL RÖSSLER OSCILLATORS}

Systems of two interacting period-doubling oscillators have been investigated by a significant number of authors, often with a view on the stability problems associated with chaos-chaos synchronization and the application of chaotic oscillators for secure communication. ${ }^{43-45}$ Pecora et al., ${ }^{45}$ for instance, present several widely used coupling configurations, provide examples of their realization in terms of electronic circuits, and discuss their individual strengths and weaknesses. Anishchenko et al. ${ }^{46}$ show sketches of bifurcation diagrams for unidirectionally and symmetrically coupled nonlinear electronic oscillators. Rasmussen et al. ${ }^{47}$ provide detailed one- and two-dimensional bifurcation diagrams for a system of two symmetrically coupled Rössler oscillators, demonstrating, among other results, how new saddle-node bifurcation curves emerge from the perioddoubling curves in an alternating manner.

As mentioned above, multi-dimensional systems that display period-doubling cascades along the edge of a synchronization tongue typically exhibit the special scaling behavior known as C-type criticality. ${ }^{26,27}$ The bifurcation structure observed for such systems differs essentially from the structures we know from low-dimensional systems. Until now, however, few examples of this transition have been worked out for realistic systems. A recent study of the C-type transition for a single-nephron model subjected to a periodic variation of the arterial pressure ${ }^{28}$ has confirmed the theoretical predictions for this kind of system and has also lead to a more detailed picture of the involved bifurcation structure. However, the structure we observe in the coupled nephron model is different in that the transition to synchronization occurs through the combination of a homoclinic and a torus bifurcation.

In order to suggest a more common character of these results and establish the role of the different bifurcations, let us consider the much simpler system of two coupled, nonidentical Rössler oscillators,

$$
\begin{aligned}
& \dot{x}_{1}=-y_{1}-z_{1} \\
& \dot{y}_{1}=x_{1}+a_{1} y_{1} \\
& \dot{z}_{1}=b+z_{1}\left(x_{1}-c_{1}\right)+d\left(z_{2}-z_{1}\right) \\
& \dot{x}_{2}=-\omega y_{2}-z_{2} \\
& \dot{y}_{2}=\omega x_{2}+a_{2} y_{2} \\
& \dot{z}_{2}=b+z_{2}\left(x_{2}-c_{2}\right)+d\left(z_{1}-z_{2}\right),
\end{aligned}
$$

where $a_{2}=0.345, b=2, c_{1}=4, c_{2}=3.9$, and the coupling parameter $d=0.1$. The value of $a_{2}$ is chosen such that the second oscillator in the uncoupled state operates near the point of its first period doubling. The gain parameter $a_{1}$ of the first oscillator and the mistuning (relative frequency) $\omega$ are used as control parameters. The difference between $c_{1}$ and $c_{2}$ represents a built-in asymmetry introduced to avoid results that depend on the precise symmetry between the two oscillators.

Figure 6 provides a survey of the region of existence of different stable modes in and around the main resonance zone for the coupled Rössler oscillators. Different shades of blue and green are again used to distinguish the low-periodic stable cycles that arise in the initial steps of the perioddoubling cascade. Brown denotes quasiperiodic dynamics, and orange denotes deterministic chaos. Gray identifies the region of existence for phase synchronized chaos. Black curves represent saddle-node bifurcations and red curves are torus bifurcation curves. Yellow and white curves are period doubling bifurcation curves for the period-1 and period-2 cycles, respectively. In analogy with Fig. 2, parts of the period-doubling curves relate to transitions that involve unstable cycles. In that case there is no change in the stable mode, and the color of the chart remains unchanged.

The first period-doubling transition takes a somewhat unusual form in that it (in the brown triangular region in the lower right corner of the resonance zone) occurs via the combination of a torus bifurcation (red curve) and a saddle-node bifurcation (black curve). As demonstrated by Reick and Mosekilde, ${ }^{48}$ the dissipative coupling of two similar perioddoubling systems leads to the replacement of the first perioddoubling bifurcation for the uncoupled Rössler oscillator by a torus bifurcation in the coupled system. This replacement arises from a splitting of the bifurcations that occur in the individual systems into sets of symmetric (in-phase) and antisymmetric (anti-phase) transitions with a subsequent destabilization of the anti-phase period- 1 cycle in a torus bifurcation, and the birth of the anti-phase period- 2 cycle in a saddle-node

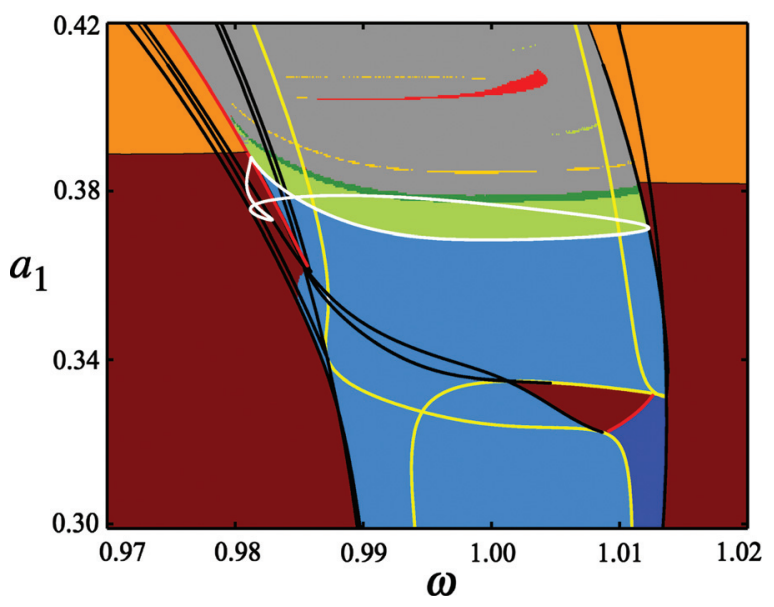

FIG. 6. (Color) Overview of the bifurcation structure of the 1:1 resonance zone for a pair of coupled, non-identical Rössler oscillators. Different shades of blue and green denote low-periodic cycles in the period-doubling cascade, yellow represents period-3 dynamics, and brown represents quasiperiodic dynamics. Gray and orange represent phase synchronized and nonsynchronized chaos, respectively. Note the large number of saddle-node bifurcation curves that accumulate along the left hand side of the resonance region. 
bifurcation. However, like the subsequent period-doubling bifurcations along the right hand side of the resonance regime, it follows the general structure for C-type criticality. In particular, we note the formation of a new saddle-node bifurcation curve along the edge of the resonance zone.

Similar saddle-node bifurcation curves are generated at each level in the period-doubling cascade along the right hand side of the resonance region. As previously noted, they serve to delineate the ranges of existence for the resonance cycles born in the period-doubling bifurcations. Previously generated saddle-node bifurcation curves continue to exist to delineate the range of existence for the now unstable resonance cycles of lower periodicity. ${ }^{47}$ The new saddle-node bifurcation curves emerge from a point on the corresponding period-doubling curve a little away from the point at which the former saddle-node bifurcation curve is tangent to the period-doubling curve.

From the triangular region of torus dynamics associated with the first period-doubling transition, two saddle-node bifurcation curves proceed all the way to the left hand edge of the resonance tongue to interact with the saddle-node bifurcation generated from the yellow period-doubling curve at this side of the resonance zone. As we shall see, this phenomenon is reflected in the generation of three saddle-node bifurcation curves for each pair of subsequent period-doubling bifurcations at the left hand side of the resonance zone.

More interesting, however, is the observation that each pair of period-doubling bifurcations give rise to the generation of a torus bifurcation curve that extends along the boundary of the left hand side of the resonance zone. A similar formation of torus bifurcation curves does not occur along the right hand edge of the resonance tongue. In this way, the bifurcation structure for the right hand side of the resonance tongue for the coupled Rössler systems corresponds to that of the upper part of the resonance zone for our coupled nephron model, and the bifurcation structure of the left hand side of the resonance zone for the coupled Rössler systems corresponds to the structure observed in the lower part of the resonance zone for the coupled nephrons. The difference between the two sides of the resonance tongue reflects the lack of symmetry between the two interacting oscillators.

To pursue these observations a little further, Fig. 7(a) shows a more detailed bifurcation diagram for the first pair of interconnected period-doubling bifurcations near the left hand side of the resonance zone. The period-doubling curves are green, the saddle-node bifurcation curves black, and the torus bifurcation curves red. For the period-doubling curves, we distinguish between fully drawn curves, dashed curves, and dotted curves depending on whether a stable node, respectively, a saddle cycle with one or two unstable directions is involved in the period-doubling transition.

When inspecting Fig. 7(a), we can follow the perioddoubling curve $P D_{2}^{S}$ for the stable period-2 cycle from the right hand side of the figure to the point $C_{2}$ where the torus bifurcation curve $T_{2}$ intersects with $P D_{2}^{S}$ and the next torus bifurcation $T_{4}$ is born. From this point $P D_{2}$ continues as a curve of period-doubling for doubly-unstable period- 2 cycles until it reaches the intermediate saddle-node bifurcation curve $S N_{2}^{b}$. From here, the curve continues first to the inner-
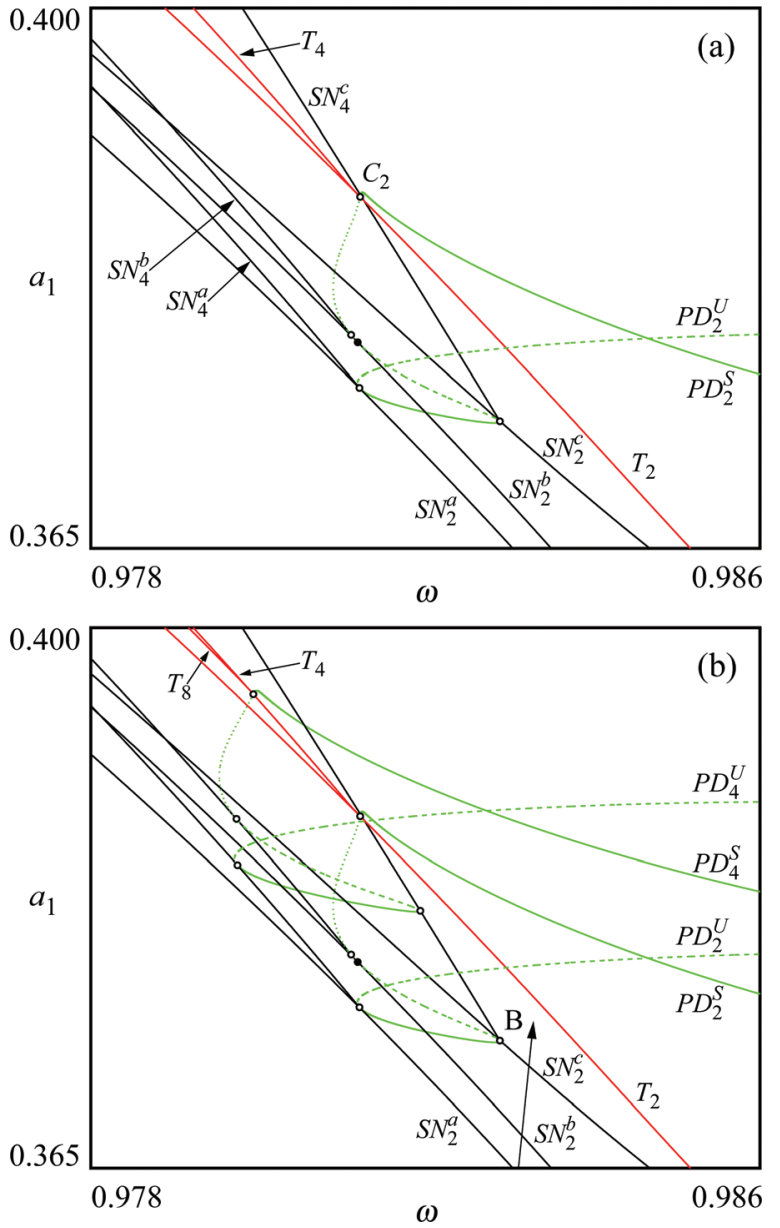

FIG. 7. (Color) Detail of the bifurcation diagram in Fig. 6 illustrating the bifurcations that take place close to the lower left zone boundary. (a) Bifurcation structure for the period-2 cycles. (b) Bifurcation structure for the period- 2 and period- 4 cycles. Note the birth of three new saddle-node bifurcation curves and one new torus bifurcation curve for each level in the period-doubling cascade.

most saddle-node bifurcation curve $S N_{2}^{c}$ as a period-doubling curve for singly unstable saddle cycles and thereafter to the outmost saddle-node bifurcation curve $S N_{2}^{a}$ as a perioddoubling curve for stable node period-2 resonant cycles.

The period- 4 cycles produced at the period-doubling curves require their own system of saddle-node and torus bifurcation curves to restrict their ranges of existence. Hence, close to (but not in) each of the points where the perioddoubling bifurcation curve is tangent to one of the saddlenode bifurcation curves $S N_{2}^{a}, S N_{2}^{b}$, and $S N_{2}^{c}$ delineating the ranges of existence for the various resonant period- 2 cycles, new saddle-node bifurcation curves are born to delineate the range of existence for the period-4 node, saddle, and doublyunstable saddle cycles. Similarly, at the point $C_{2}$ where the period-doubling curve $P D_{2}^{S}$ intersects the torus bifurcation curve $T_{2}$, a new torus bifurcation curve $T_{4}$ is born.

As shown in Fig. 7(b), precisely the same picture applies to the period-doubling curves for the period- 4 cycles and to the following curves in the period-doubling transitions as well. We conclude that the system generates a set of three saddle-node bifurcation curves for each pair of perioddoublings along the edge of the resonant domain. At the same time, the system gives birth to a new torus bifurcation 
curve and, as our preliminary investigation shows, this set of torus bifurcation curves accumulate in an alternating manner along the boundary of the resonance zone.

To provide a clear picture of the different steps in the transition from ergodic (quasiperiodic) dynamics to synchronous periodic dynamics, Fig. 8(a) shows a one-dimensional bifurcation diagram obtained along the direction $B$ in Fig. 7(b). To the left in this diagram, we observe the ergodic torus that exists outside of the resonance zone. As this torus approaches the resonance zone near the range of period- $2 \mathrm{dy}-$ namics, it first undergoes a torus doubling bifurcation. ${ }^{29,31}$ The close-up of the upper branch of the torus displayed in Fig. 8(b) shows that the saddle-node bifurcations at $S N_{2}^{a}, S N_{2}^{b}$, and $S N_{2}^{c}$ all take place in a regime where the produced stable and unstable period- 2 cycles exist together with the stable period2 torus. This regime may be considered as a satellite structure to the main synchronization regime, a structure in which synchronized and non-synchronous dynamics co-exist and where additional bifurcations may occur.

Immediately to the left of the torus bifurcation point $T_{2}$, the larger amplitude period-doubled torus is replaced by a phase synchronized period-2 torus of much smaller amplitude. This transition involves a homoclinic bifurcation. In
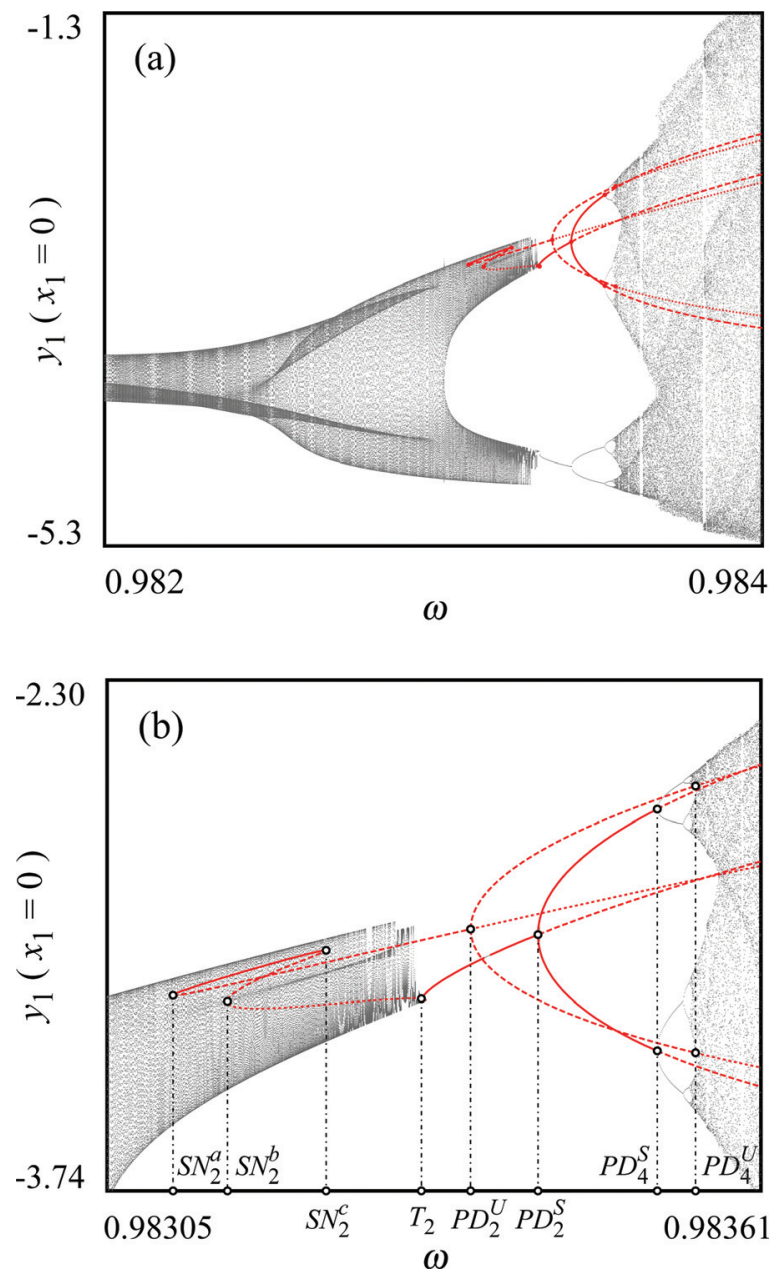

FIG. 8. (Color) (a) One-dimensional bifurcation diagram along the direction $B$ in Fig. 7(b). Note the period-doubling of the ergodic torus in the left hand side of the figure. (b) Close-up on the upper branch of (a). The stable resonant mode arises in a reverse torus bifurcation at $T_{2}$ rather than in a saddlenode bifurcation. the region of the small phase synchronized torus, the two Rössler systems display quasiperiodic dynamics, but the variation in their phase difference remains less than $2 \pi$. At $T_{2}$, the phase synchronized torus disappears in a reverse torus bifurcation and produces a stable period-2 (focus) cycle. This cycle soon transforms into a stable node, and to the right in the bifurcation diagram, we can follow how the stable period-2 cycle together with a saddle period- 2 cycle originating in the above saddle-node bifurcations undergo interconnected cascades of period-doubling bifurcations. The transition from quasiperiodicity to synchronized periodic dynamics thus takes place via a torus bifurcation. In a small interval to the right of $T_{2}$, like for the coupled nephron system, one observes the phenomenon of phase synchronized quasiperiodic dynamics.

\section{CONCLUSIONS}

Synchronization of different forms of oscillatory dynamics plays an essential role in the functioning and regulation of physiological systems, ${ }^{1}$ from cell-to-cell communication to the mutual adjustment of hormonal release processes ${ }^{7}$ and the interaction of organs. ${ }^{2}$ Both excessive and insufficient synchronization of cellular activity in different tissues may be the causal factor for the development of a disease. ${ }^{5}$

However, although the classic theory of synchronization has been useful to interpret a broad range of observations in biology as well as in other fields of science, physiological control systems display so many different and mutually interacting nonlinear dynamic processes that more complicated phenomena should be expected. The investigation of phase synchronized chaos ${ }^{39,40}$ in periodically forced perioddoubling systems has lead to development of a scaling theory for a period-doubling transition that unfolds along the edge of a synchronization tongue. ${ }^{26,27}$ However, a comprehensive description of the synchronization mechanisms in systems of coupled period-doubling oscillators appears not previously to have been presented, and many problems also remain to be solved with respect to the synchronization of systems with two or more internal modes of oscillation.

Experimental evidence from rats collected over more than two decades has shown that the individual functional unit (nephron) of the kidney makes use of two different and mutually interacting oscillatory mechanisms to regulate the incoming blood flow and that these oscillatory processes move in and out of different states of synchronization. ${ }^{10,11}$ For normotensive rats, about $50 \%$ of the experimental time series for the proximal tubular pressure shows the episodes of period-doubling dynamics, ${ }^{20}$ and for hypertensive rats the time series are generally found to be chaotic. ${ }^{33}$ The individual nephron thus represents a two-mode oscillator that undergoes a period-doubling transition.

Nephrons are typically arranged in pairs (or triplets) that share part of an afferent arteriole and, therefore, display a particularly strong interaction. ${ }^{21}$ This interaction causes them to synchronize their tubular pressure variations for periods of perhaps 15-30 min, lose synchrony, and then reestablish synchronization in the same or a new pattern. Both the slow TGFmediated and the faster myogenic modes can synchronize 
alone, but it is also possible that the two modes simultaneously synchronize between two adjacent nephrons. ${ }^{15,16} \mathrm{~A}$ recent study, ${ }^{49}$ in which laser speckle microscopy was applied to simultaneously detect the TGF-mediated oscillations (in the efferent arteriolar blood flow) for a large group of superficial nephrons, has shown how clusters of synchronized nephrons arise and disappear in a complex dynamical pattern reflecting partly the underlying vascular structure, but also supporting the view of the continuous transition between synchronous and asynchronous motions as an element in the normal regulation of the kidney.

Using a well-documented model of a pair of vascularly coupled nephrons, the present paper established a detailed description of the bifurcation structure for a system of two interacting, nearly identical period-doubling systems. Our main observations are:

- Cascades of saddle-node bifurcations are generated along both sides of the synchronization region. These saddlenode bifurcations serve to delineate the region of synchronized periodic dynamics, and each new perioddoubling bifurcation is associated with the birth of a new set of saddle-node bifurcation curves. The saddle-node bifurcation curves emerge alternately from the stable and the unstable branch of the corresponding period-doubling curve, but always a little away from the point at which the period-doubling curve is tangent to the former saddle-node bifurcation curve.

- The two sides of the synchronization region are different, however. On one side the system follows the bifurcation structure known for periodically forced period-doubling systems (C-type criticality ${ }^{26,27}$ ). Along the other edge, the system displays a new and somewhat more complex structure.

- The difference between the two sides of the synchronization regime arises from the (slight) asymmetry between the two interacting nephrons. Coupling of two nearly identical period-doubling oscillators leads to a splitting of the bifurcations observed in the individual oscillator into symmetric and anti-symmetric bifurcations. The symmetric bifurcations are associated with in-phase motion of the two oscillators and the anti-symmetric bifurcations with anti-phase motion. When the oscillators are different, as assumed here, the two sets of transitions tend to manifest themselves on either side of the resonance zone.

- With this new bifurcation structure, the stable periodic resonance mode disappears via a torus bifurcation. This bifurcation is unusual by the fact that it produces a behavior in which the two nephrons move in a phase synchronized quasiperiodic mode. A torus bifurcation cannot occur for the symmetric modes, but it is a natural bifurcation after an anti-symmetric period-doubling bifurcation. Each torus bifurcation curve is accompanied by a curve of homoclinic bifurcations, and the synchronized quasiperiodic motion finally disappears in a bifurcation of this type.

To demonstrate that the structure observed for two coupled nephrons has a broader range of occurrence for interacting period-doubling systems, we have shown that a similar structure arises in a system of two coupled Rössler oscillators. The Rössler oscillator may be considered the archetype of a time-continuous period-doubling oscillator, and demonstrating that a particular phenomenon arises in a system of coupled Rössler oscillators is likely to imply that the same phenomenon will occur in a class of similarly coupled period-doubling systems in cell biology, physiology, chemistry, physics, etc.

\section{ACKNOWLEDGMENTS}

We would like to thank S. P. Kuznetsov for clarifying discussions about C-type criticality. A. N. Pavlov has provided the power spectrum in Fig. 1(c).

${ }^{1}$ J. Bélair, L. Glass, U. an der Heiden, and J. Milton, Chaos 5, 1 (1995).

${ }^{2}$ C. Schäfer, M. G. Rosenblum, J. Kurths, and H.-H. Abel, Nature (London) 392, 239 (1998).

${ }^{3}$ R. Bertram, J. Previte, A. Sherman, T. A. Kinard, and L. S. Satin, Biophys. J. 79, 2880 (2000).

${ }^{4}$ J. C. B. Jacobsen, C. Aalkjaer, V. V. Matchkov, H. Nilsson, J. J. Freiberg, and N.-H. Holstein-Rathlou, Philos. Trans. R. Soc. A 366, 3483 (2008).

${ }^{5}$ U. B. Barnikol, O. V. Popovych, C. Hauptmann, V. Sturm, H.-J. Freund, and P. A. Tass, Philos. Trans. R. Soc. A 366, 3545 (2008).

${ }^{6}$ J. Modolo, B. Bhattacharya, R. Edwards, J. Campagnaud, A. Legros, and A. Beuter, Front. NeuroSci. 4, 45 (2010).

${ }^{7}$ F. Kronenberg, L. J. Cote, D. M. Linkie, I. Dyrenfurth, and J. A. Downey, Maturitas 6, 31 (1984).

${ }^{8}$ F. Lévi, A. Altinok, J. Clairambault, and A. Goldbeter, Philos. Trans. R. Soc. A 366, 3575 (2008).

${ }^{9}$ N.-H. Holstein-Rathlou and P. P. Leyssac, Acta Physiol. Scand. 126, 333 (1986).

${ }^{10}$ N.-H. Holstein-Rathlou, Pflügers Arch. 408, 438 (1987).

${ }^{11}$ N.-H. Holstein-Rathlou and D. J. Marsh, Physiol. Rev. 74, 637 (1994).

${ }^{12}$ H. Gustafsson, A. Bulow, and H. Nilsson, Acta Physiol. Scand. 152, 145 (1994).

${ }^{13}$ M. J. Davis and M. A. Hill, Physiol. Rev. 79, 387 (1999).

${ }^{14}$ N.-H. Holstein-Rathlou, A. J. Wagner, and D. J. Marsh, Am. J. Physiol. 260, F53 (1991).

${ }^{15}$ O. V. Sosnovtseva, A. N. Pavlov, E. Mosekilde, and N.-H. Holstein-Rathlou, Phys. Rev. E 66, 61909-1-7 (2002).

${ }^{16}$ O. V. Sosnovtseva, E. Mosekilde, A. N. Pavlov, N.-H. Holstein-Rathlou, and D. J. Marsh, Phys. Rev. E 70, 031915-1-8 (2004).

${ }^{17}$ E. VanBavel, E. N. Bakker, A. Pistea, O. Sorop, and J. A. Spann, Clin. Hemorheol. Microcirc. 34, 35 (2006).

${ }^{18}$ L. C. Moore, Am. J. Physiol. 247, F267 (1984).

${ }^{19}$ R. Feldberg, M. Colding-Jorgensen, and N.-H. Holstein-Rathlou, Am. J. Physiol. 269, F581 (1995).

${ }^{20}$ A. N. Pavlov, O. V. Sosnovtseva, O. N. Pavlova, E. Mosekilde, and N.-H. Holstein-Rathlou, Chaos, Solitons Fractals 41, 930 (2009).

${ }^{21}$ D. Casellas, M. Dupont, N. Boriquet, L. C. Moore, A. Artuso, and A. Mimran, Am. J. Physiol. 267, F931 (1994).

${ }^{22}$ A. J. Wagner, N.-H. Holstein-Rathlou, and D. J. Marsh, Am. J. Physiol. 272, F372 (1997).

${ }^{23}$ N.-H. Holstein-Rathlou, K.-P. Yip, O. V. Sosnovtseva, and E. Mosekilde, Chaos 11, 417 (2001).

${ }^{24}$ D. J. Marsh, O. V. Sosnovtseva, E. Mosekilde, and N.-H. Holstein-Rathlou, Chaos 17, 15114-1-10 (2007).

${ }^{25}$ V. I. Arnold, Am. Math. Soc. Transl. Ser. 2 46, 213 (1965).

${ }^{26}$ S. P. Kuznetsov, Phys. Rev. E 72, 026205 (2005).

${ }^{27}$ S. P. Kuznetsov, A. P. Kuznetsov, and I. R. Sataev, J. Stat. Phys. 121, 697 (2005).

${ }^{28}$ J. L. Laugesen, E. Mosekilde, and N.-H. Holstein-Rathlou, J. R. Soc. Interface Focus 1, 132 (2011).

${ }^{29}$ A. Arnéodo, P. H. Coullet, and E. A. Spiegel, Phys. Lett. A 94, 1 (1983).

${ }^{30}$ K. Kaneko, Prog. Theor. Phys. 72, 202 (1984).

${ }^{31}$ M. Sekikawa, N. Inaba, T. Yoshinaga, and T. Tsubouchi, Phys. Lett. A 348, 187 (2006).

${ }^{32}$ N.-H. Holstein-Rathlou, J. Am. Soc. Nephrol. 4, 1275 (1993).

${ }^{33}$ M. Barfred, E. Mosekilde, and N.-H. Holstein-Rathlou, Chaos 6, 280 (1996).

${ }^{34}$ J. L. Laugesen, O. V. Sosnovtseva, E. Mosekilde, N.-H. Holstein-Rathlou, and D. J. Marsh, Am. J. Physiol. 298, R997 (2010). 
${ }^{35}$ D. E. Postnov, O. V. Sosnovtseva, and E. Mosekilde, Chaos 15, 013704 (2005).

${ }^{36}$ P. P. Leyssac and N.-H. Holstein-Rathlou, Pflügers Arch. 413, 267 (1989).

${ }^{37}$ Y. A. Kuznetsov, Applied Mathematical Sciences: Elements of Applied Bifurcation Theory (Springer-Verlag, New York, 2004).

${ }^{38}$ E. J. Doedel, R. C. Paffenroth, A. R. Champneys, T. F. Fairgrieve, Y. A. Kuznetsov, B. E. Oldeman, B. Sandstede, and X. J. Wang, Technical Report (Available via ftp from ftp.cs.concordia.ca/pub/doedel/auto.), California Institute of Technology, Pasadena CA91125 (2000).

${ }^{39}$ M. Rosenblum, A. Pikovsky, and J. Kurths, Phys. Rev. Lett. 76, 1804 (1996).

${ }^{40}$ A. Pikovsky, M. Rosenblum, and J. Kurths, Synchronization, (Cambridge University Press, Cambridge, 2001).

${ }^{41}$ V. Anishchenko, S. Nikolaev, and J. Kurths, Phys. Rev. E 73, 056202 (2006).
${ }^{42}$ V. Anishchenko, S. Nikolaev, and J. Kurths, Phys. Rev. E 76, 046216 (2007).

${ }^{43}$ N. F. Rulkov and M. M. Sushchik, Int. J. Bifurcation Chaos 7, 625 (1997).

${ }^{44}$ S. Yanchuk, Yu. Maistrenko, and E. Mosekilde, Physica D 154, 26 (2001).

${ }^{45}$ L. M. Pecora, T. L. Carroll, G. A. Johnson, and D. J. Mar, Chaos 7, 520 (1997).

${ }^{46}$ V. S. Anishchenko, T. E. Vadivasova, D. E. Postnov, and M. A. Safonova, Int. J. Bifurcation Chaos 2, 633 (1992).

${ }^{47}$ J. Rasmussen, E. Mosekilde, and C. Reick, Math. Comput. Simul. 40, 247 (1996).

${ }^{48}$ C. Reick and E. Mosekilde, Phys. Rev. E 52, 1418 (1995).

${ }^{49}$ N.-H. Holstein-Rathlou, O. V. Sosnovtseva, A. N. Pavlov, W. A. Cupples, C. M. Sørensen, and D. J. Marsh, Am. J. Physiol. Ren. Physiol. 300, F31929 (2011). 